\title{
Sequencing of transcriptomes from two Miscanthus species reveals functional specificity in rhizomes, and clarifies evolutionary relationships
}

\author{
Changsoo Kim ${ }^{1 \dagger}$, Tae-Ho Lee ${ }^{1,4 \dagger}$, Hui Guo ${ }^{1}$, Sung Jin Chung², Andrew H Paterson', Do-Soon Kim³ \\ and Geung-Joo Lee 2* $^{*}$
}

\begin{abstract}
Background: Miscanthus is a promising biomass crop for temperate regions. Despite the increasing interest in this plant, limited sequence information has constrained research into its biology, physiology, and breeding. The whole genome transcriptomes of $M$. sinensis and $M$. sacchariflorus presented in this study may provide good resources to understand functional compositions of two important Miscanthus genomes and their evolutionary relationships.

Results: For M. sinensis, a total of 457,891 and 512,950 expressed sequence tags (ESTs) were produced from leaf and rhizome tissues, respectively, which were assembled into 12,166 contigs and 89,648 singletons for leaf, and 13,170 contigs and 112,138 singletons for rhizome. For M. sacchariflorus, a total of 288,806 and 267,952 ESTs from leaf and rhizome tissues, respectively, were assembled into 8,732 contigs and 66,881 singletons for leaf, and 8,104 contigs and 63,212 singletons for rhizome. Based on the distributions of synonymous nucleotide substitution (Ks), sorghum and Miscanthus diverged about 6.2 million years ago (MYA), Saccharum and Miscanthus diverged 4.6 MYA, and $M$. sinensis and M. sacchariflorus diverged 1.5 MYA. The pairwise alignment of predicted protein sequences from sorghum-Miscanthus and two Miscanthus species found a total of 43,770 and 35,818 nsSNPs, respectively. The impacts of striking mutations found by nsSNPs were much lower between sorghum and Miscanthus than those between the two Miscanthus species, perhaps as a consequence of the much higher level of gene duplication in Miscanthus and resulting ability to buffer essential functions against disturbance.
\end{abstract}

Conclusions: The ESTs generated in the present study represent a significant addition to Miscanthus functional genomics resources, permitting us to discover some candidate genes associated with enhanced biomass production. Ks distributions based on orthologous ESTs may serve as a guideline for future research into the evolution of Miscanthus species as well as its close relatives sorghum and Saccharum.

Keywords: Miscanthus sinensis, Miscanthus sacchariflorus, Expressed sequence tags, Synonymous substitution, Orthologous sequences, Nonsynonymous SNPs

\section{Background}

Concerns about global warming in combination with increased use of fossil fuel have spurred growing interest in sources of renewable energy such as biofuels. The genus Miscanthus has been considered attractive as a feedstock for cellulosic biofuel production because the plants are adapted to temperate latitudes yet utilize the

\footnotetext{
* Correspondence: gjlee@cnu.ac.kr

'Equal contributors

${ }^{2}$ Department of Horticulture, Chungnam National University, Daejeon

305-764, South Korea

Full list of author information is available at the end of the article
}

energy-efficient $\mathrm{C} 4$ photosynthetic pathway, producing high yields of biomass but with low-nutrient requirements, adaptation to marginal land due to resistance to abiotic stress, and not competing with use for food [1-3]. At present, 14 Miscanthus species are recognized, most of which are native to eastern Asia, but with a few found in Polynesia, the Himalayas, and southern Africa [4].

Miscanthus belongs to the Andropogoneae tribe of grasses, including many economically important crops such as maize, sorghum, and sugarcane. In particular, Miscanthus and sugarcane belong to the subtribe 
Saccharinae that may be characterized by its complex and high ploidy genomes. For example, $M . \times$ giganteus $(2 n=3 \times=57)$, which is specifically of interest due to its ability to accumulate biomass, is thought to result from crosses between $M$. sacchariflorus $(2 \mathrm{n}=4 \times=76)$ and $M$. sinensis $(2 \mathrm{n}=2 \times=38)$ [5]. Study of the large and complex Miscanthus genomes is challenging, although the whole genome sequence of closely-related sorghum $(2 \mathrm{n}=2 \times=20)$ [6] provides a valuable reference for molecular research in Miscanthus. Genomic study of Miscanthus is currently focused on the construction of genetic maps using AFLP [7], SSR [8], RNA-seq [9], and GBS (genotype by sequencing) [10].

Expressed sequence tags (ESTs) are frequently useful for plant species that do not have sufficient genomics resources, providing functional profiles of genes as well as a basis of evolutionary study. Owing to massively parallel sequencing technologies, large numbers of ESTs can be easily generated under different growing conditions or tissue types; however, only five Miscanthus ESTs are publicly available in the NCBI's dbEST as of December, 2013 (http://www.ncbi.nlm.nih.gov/dbEST).

In the current study, we generated transcriptome data sets from $M$. sacchariflorus and M. sinensis, the suspected progenitors of the $M . \times$ giganteus species which is preferred for biomass production. In particular, we chose those two species to scan transcriptomes because they will be served as mapping parents and the collection of gene profile may be necessary in the future work. We determined functional profiles of transcriptome sets from both leaf and rhizome for each Miscanthus species and compared the profiles. M. sinensis is known to have weak (or sometimes no) rhizomes but M. sacchariflorus has vigorous rhizomes, so it was of interest to identify genes showing significantly different expression in rhizomes than other tissues [11]. In addition, using the massive transcriptome data, we estimated the time of divergence among the two Miscanthus species, sorghum and Saccharum.

\section{Results and discussion}

454 sequencing and de novo assembly of Miscanthus EST In $M$. sinensis, a total of 457,891 and 512,950 reads from leaf and rhizome cDNA libraries were generated, spanning about 140 mega base pairs (Mbps) and $155 \mathrm{Mbps}$, respectively. The numbers of reads from the leaf $(288,806)$ and rhizome $(267,952)$ libraries in $M$. sacchariflorus were slightly less than those in $M$. sinensis; however, the average read length was longer than in $M$. sinensis (Table 1). After the assembly of raw reads (Materials and Methods for details), the leaf (MSIL, hereafter) and rhizome (MSIR) cDNA libraries of $M$. sinensis generated a total of 12,166 and 13,170 contigs, respectively. The leaf (MSAL) and rhizome (MSAR) libraries in M. sacchariflorus have 8,731 and 8,104 contigs, respectively. It is no surprise that the number of reads is positively correlated with that of contigs. The average numbers of reads in a contig are 28.4, 28.6, 23.6, and 23.4 for MSIL, MSIR, MSAL, and MSAR, respectively. The length distribution of the contigs is shown in Figure 1. The assembly produced a substantial number of large contigs in M. sinensis $(12,144$ for leaf and 13,146 for rhizome were $\geq 200 \mathrm{bp}$ in length). Likewise, 8,721 and 8,095 contigs were assembled to more than $200 \mathrm{bp}$ in MSAL and MSAR, respectively. The average contig lengths were 923 (MSI) and 993 bp (MSA) while the average singleton lengths were 281 (MSI) and $303 \mathrm{bp}$ (MSA). The genome-wide coverage of unigenes based on the size of the haploid genome of M. sinensis and $M$. sacchariflorus [12] was about $1.5 \%$ and $1.1 \%$, respectively, while the estimated coverage of coding sequences in sorghum was $5.5 \%$ [6]. However, genome size is not consistent with the number of genes (C-value paradox). The haploid genome size of Miscanthus spp. is about three times bigger than that of sorghum, in part due to the whole genome duplication event after its divergence from the sorghum lineage [8-10]; thus, simple comparison of coding sequences to each genome may not be able to explain the coverage of our ESTs to total transcriptomes in Miscanthus. For example, a total of 34,355 (MSI) and 35,177 (MSA) unigenes (out of contigs and singletons combined in Table 1) have significant similarities to sorghum gene models $(34,496$ in total) [6] based on blast search $\left(e<10^{-5}\right)$, indicating that the number of Miscanthus ESTs presented in this study is sufficient for further analysis.

\section{Functional annotation of ESTs}

In order to assign putative functions to ESTs, only contigs were subjected to similarity searches because short singletons could yield false positive results. First, contigs were blasted against all the genome sequences in major biological databases such as Phytozome [13] and KOG (euKaryotic Orthologous Group) [14] with a cutoff e-value of $10^{-5}$ (Figure 2). A total of $93 \%(11,377)$ of the total MSIL contigs and $81 \%(10,733)$ of the total MSIR contigs matched Phytozome transcripts from one or more of twenty-five plant species, while $94 \%$ of MSAL and MSAR contigs had significant hits. Contigs from rhizomes had relatively fewer matches (the number of hits in a specific database/total number of contigs obtained from Miscanthus) than those from leaves, consistent with limited sequence information available from rhizomatous plants and limited knowledge of rhizome-specific gene functions. Next, we blasted the contigs against the KOG in order to determine conserved orthologous genes. MSIL and MSIR contigs had $56 \%$ and $54 \%$ significant hits against the KOG versus $58 \%$ and $55 \%$ for MSAL and MSAR, respectively. M. sacchariflorus shows higher proportions than $M$. sinensis because of the smaller number of contigs. In addition, since 
Table 1 Sequencing and assembly statistics for Miscanthus sinensis and M. sacchariflorus ESTs

\begin{tabular}{|c|c|c|c|c|}
\hline & \multicolumn{2}{|c|}{ M. sinensis } & \multicolumn{2}{|c|}{ M. sacchariflorus } \\
\hline & Leaf & Rhizome & Leaf & Rhizome \\
\hline Read No. & 457,891 & 512,950 & 288,806 & 267,952 \\
\hline Total read length (bp) & $140,496,482$ & $155,223,677$ & $96,218,568$ & $89,406,409$ \\
\hline Average read length (bp) & 307 & 303 & 333 & 333 \\
\hline Contig No & 12,166 & 13,170 & 8,732 & 8,104 \\
\hline Contig length (bp) & $11,802,712$ & $12,154,482$ & $8,670,971$ & $7,832,874$ \\
\hline Average length of contig (bp) & 970 & 923 & 993 & 967 \\
\hline Contig range (bp) & $84-5,164$ & $100-6,590$ & $125-6,402$ & $123-6,230$ \\
\hline$>=1 \mathrm{~kb}$ & $4,280(35.2 \%)$ & 4,149 (31.5\%) & $3,079(35.3 \%)$ & $2,782(34.3 \%)$ \\
\hline$>=200 \mathrm{bp}$ & 12,144 (99.8\%) & $13,146(99.8 \%)$ & $8,721(99.9 \%)$ & $8,095(99.9 \%)$ \\
\hline Singleton No. & 89,648 & 112,138 & 66,881 & 63,212 \\
\hline Singleton length (bp) & $25,195,941$ & $31,614,461$ & $20,040,968$ & $19,174,280$ \\
\hline
\end{tabular}

the KOG for plant genes was constructed solely based on Arabidopsis thaliana, the $\sim 40 \%$ of contigs which do not have significant matches to genes in the DB may reflect monocot-dicot divergence or lineage- or speciesspecific genes.

More than $50 \%$ of transcripts were specific to either leaf or rhizome libraries. Highly similar leaf and rhizome contigs were determined by BLASTN $\left(\mathrm{e}<10^{-10}\right)$ to calculate proportions of genes expressed in both organs
(Figure 3). In M. sinensis, 5,587 genes were expressed in both leaf and rhizome, comprising $45.9 \%$ and $42.4 \%$ of MSIL and MSIR, respectively. In M. sacchariflorus, 3,939 contigs were expressed in both tissues comprising $45.1 \%$ and $48.6 \%$ of MSAL $(8,732)$ and MSAR $(8,104)$, respectively.

More than $39.1 \%$ of transcripts were specific to M. sinensis or M. sacchariflorus libraries. The leaf libraries of the two Miscanthus species had 5,314 highly similar

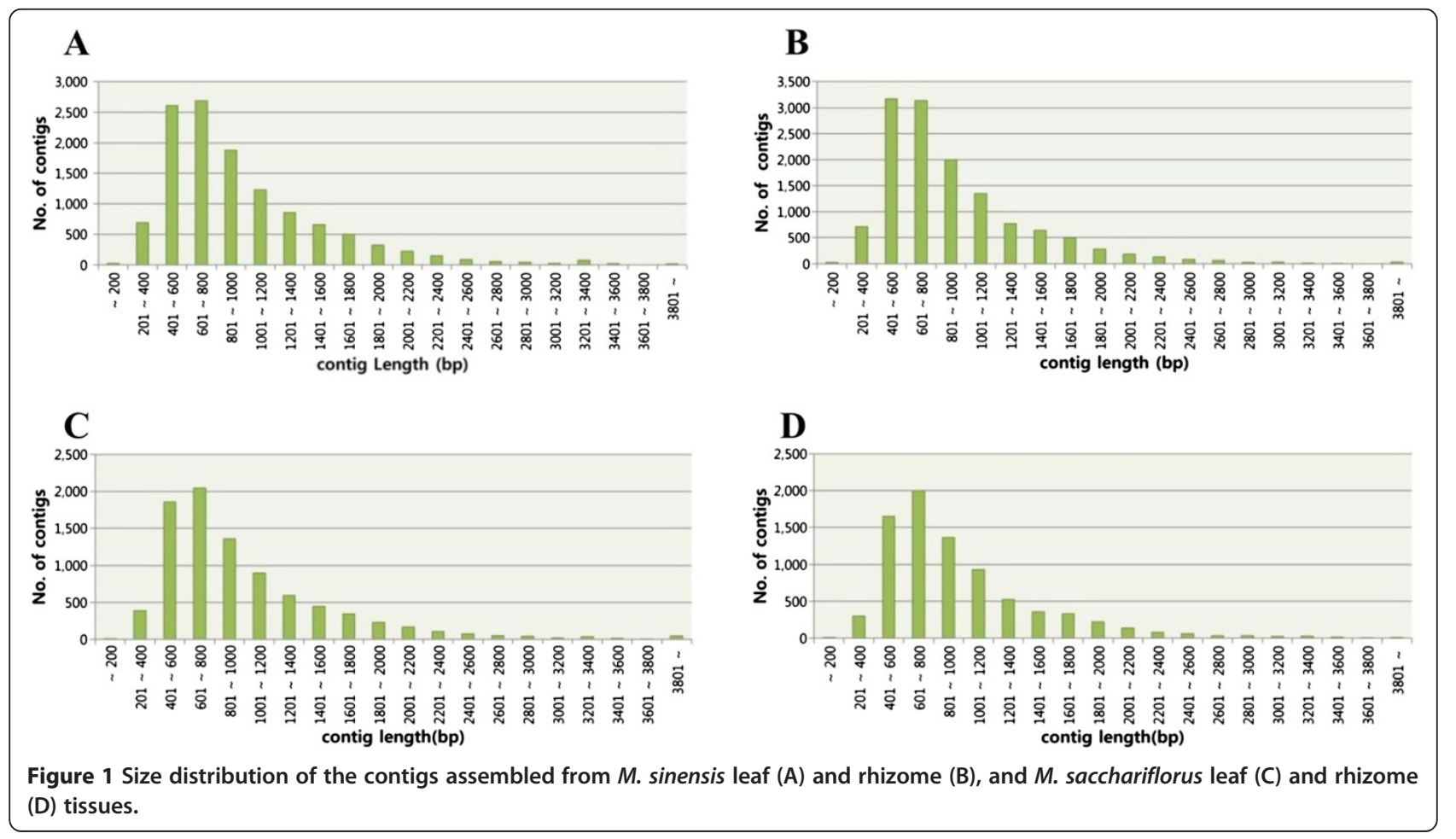




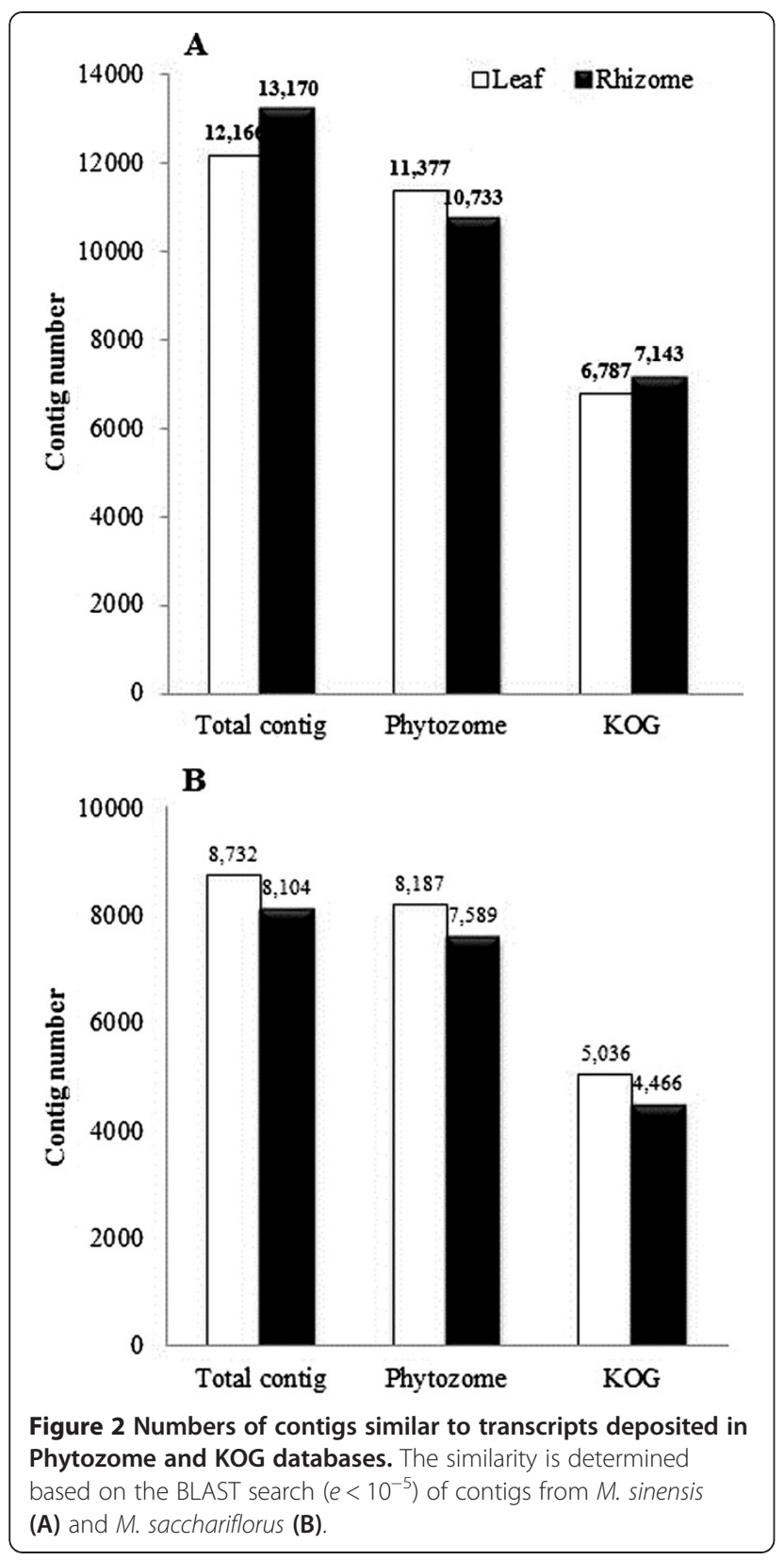

contigs which was $43.7 \%$ in MSIL and $60.9 \%$ in MSAL whereas the rhizome libraries had the 4,461 contigs whose proportion in MSIR and MSAR were $33.9 \%$ and $55.0 \%$, respectively. The relatively higher proportions of highly similar contigs between $M$. sacchariflorus and sinensis in M. sacchariflorus than in $M$. sinensis libraries might be caused by less number of contigs in $M$. sacchariflorus and that is consistent with the higher proportion of well conserved orthologous genes in M. sacchariflorus library than M. sinensis (Figure 2).

A total of 2,744 transcripts were found to be expressed in all tissue types and species. As expected, GO classification suggested that those transcripts were mostly related

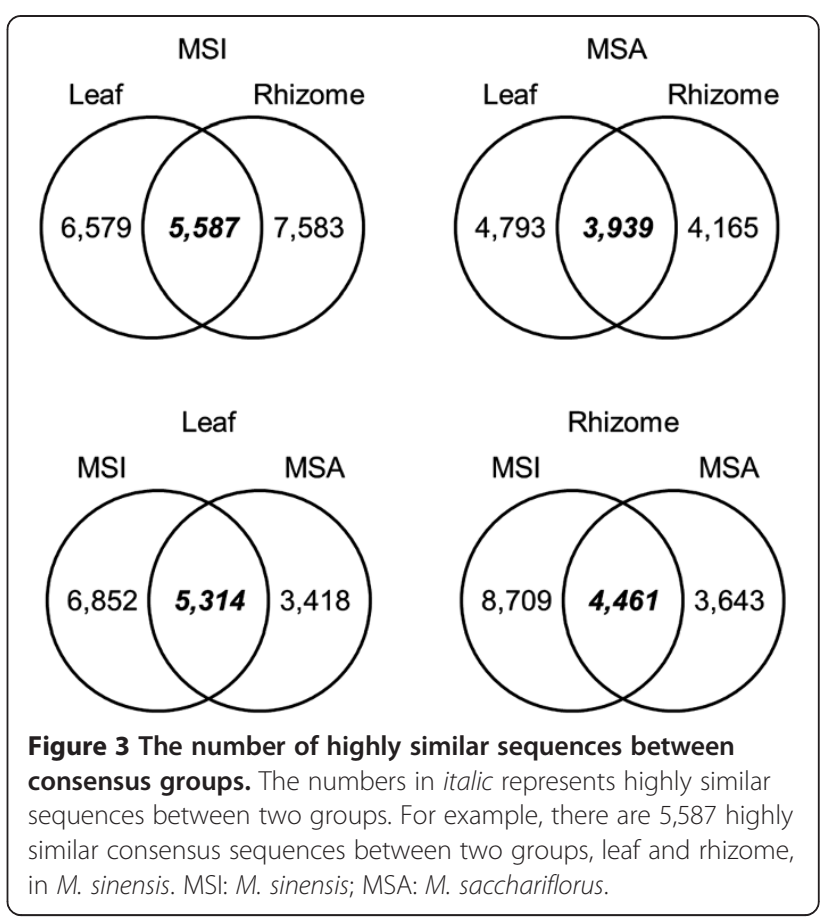

to basal plant metabolic processes or structures. Although a large number of transcripts seem to be tissue- or speciesspecific genes as shown in this work, housekeeping functions appear well conserved in different tissues and species.

\section{Comparative analysis of rhizome-enriched genes of Sorghum spp. to Miscanthus ESTs}

Previously, rhizome-enriched genes have been identified from S. halepense and S. propinquum [15]. Similarity search showed that 383 out of 768 rhizome-enriched genes found in the two Sorghum species have at least one ortholog in Miscanthus ESTs and 171 have orthologs in both leaf and rhizome from the two Miscanthus species. The 171 rhizome-enriched genes with four corresponding orthologs in Miscanthus (Sorghum orthologs in the four libraries) were defined as orthologous groups and a phylogenetic tree was generated for each orthologous group. Since $M$. sinensis is known to have weak or no rhizomes whereas M. sacchariflorus has vigorous rhizomes, only three types of trees clustering rhizome-enriched genes of the two sorghum species and rhizome ESTs of $M$. sacchariflorus (DN and AR in Figure 4, respectively) were further analyzed although 15 different topologies were generated. Interestingly, no tree topology showed clustering of rhizome-enriched genes from the two sorghum species and rhizome ESTs of $M$. sinensis, suggesting that rhizomeenriched genes from the two Sorghum species are more similar to the rhizome ESTs of M. sacchariflorus. The three topologies comprised 31 orthologous groups. Table 2 shows ESTs included in the 31 orthologous groups with their putative functions. The genes included in those three 

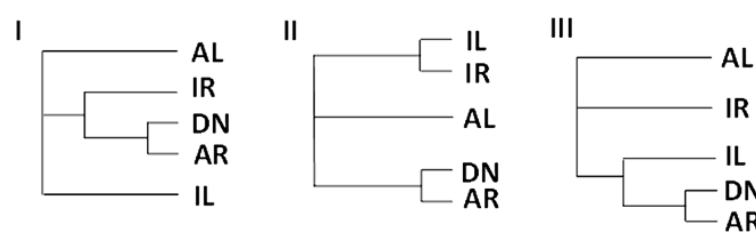

Figure 4 Phylogenetic trees in which rhizome-enriched genes of S. halepense or S. propinquum (DN) and ESTs of M. sacchariflorus (AR) are grouped together. The genes included in these trees are further analyzed as rhizome-enriched genes in Miscanthus. DN: rhizome-enriched genes of $S$. halepense or S. propinquum; IL: leaf ESTs of $M$. sinensis; IR: rhizome ESTs of $M$. sinensis; AL: leaf ESTs of M. sacchariflorus; AR: rhizome ESTs of M. sacchariflorus.

topologies were annotated by gene ontology (GO). While no particular category was predominant, the 'response to stimulus' (GO:0050896), seemingly related to signals in response to stress, occupies a high portion. Jang et al. [15] suggested that the loss of rhizomatousness in S. bicolor might have been caused by changes in gene regulation. Although the 31 genes are not specifically associated with functions and GO categories found in roots or rhizomes, these genes could be primary targets for investigating rhizomatousness in Miscanthus. The 31 genes are all expressed in both leaf and rhizome in the two Miscanthus species, but rhizome ESTs of $M$. sacchariflorus are still closer to rhizome-enriched genes from the two sorghum species, which could be caused by regulatory changes in the upstream regions of those genes. However, there are a number of ESTs exclusively expressed in Miscanthus rhizomes (Figure 3). It remains to be determined whether they are truly specific to rhizomes or due to inadequate sampling in random sequencing of transcriptomes.

\section{Speciation of Miscanthus spp., sugarcane, and sorghum}

Synonymous nucleotide substitution (Ks) of orthologous gene pairs provided insight into the timing(s) of divergence among these grasses (Figure 5). Considering $6.5 \times 10^{-9}$ synonymous changes per synonymous site per year as the neutral mutation rate in monocots [16], the divergence of Sorghinae and Saccharinae subtribes (peak A) was 0.08 , corresponding to 6.2 million years ago (MYA). Divergence between Saccharum and Miscanthus genera (peak $\mathrm{B}, \mathrm{Ks}=0.04$ ) was estimated as $4.6 \mathrm{MYA}$; and between the two Miscanthus species (peak $\mathrm{C}, \mathrm{Ks}=0.02$ ) as 1.5 MYA.

The divergence between sugarcane and sorghum has previously been investigated using orthology, with multiple studies estimating that the two species diverged about 7.7 - 9.0 MYA [3,17,18]. Since Miscanthus and sugarcane belong to the same subtribe (Saccharinae) and share a common ancestor, the estimation may be consistent with the case which sorghum and Miscanthus are compared; however, our estimation in was about $1.4-2.8$ million years more recent (6.2 MYA). The discrepancy from previous reports may be due to different sequence data used for the comparison. Kim et al. [18] and Wang et al. [3] estimated the divergence time at 7.7 MYA but Jannoo et al. [17] reported the divergence time at 8.0 - 9.0 MYA. The former two studies used sugarcane ESTs mostly originating from hybrid sugarcane cultivar(s) but the latter used only a single gene, Adh1, to estimate the divergence between the two subtribes. The sugarcane genome is more complex than Miscanthus owing to a higher ploidy number and hybrid sugarcane is still more complex because of its interspecific origin and frequent aneuploidy caused by its breeding history [19]. Therefore, although the common ancestor of Miscanthus and sugarcane diverged from the sorghum lineage at the same time, hybrid sugarcane could be highly polymorphic within single cultivars, and appear more diverged from sorghum than Miscanthus, resulting in the overestimation of Ks values. We also infer that sugarcane and Miscanthus share a common ancestor about 4.6 MYA. Due to lack of nucleotide information in Miscanthus, its divergence from the Saccharum lineage had not been estimated previously [18]. In turn, the estimation of the two genera could be overestimated because of the ESTs from hybrid sugarcane cultivar(s). However, our estimation together with EST sequences may serve as a guideline for future research in the evolution of Miscanthus species until a detail of sugarcane genome is released.

Recently published genetic maps revealed that the Miscanthus lineage experienced whole genome duplication after its divergence from sorghum $[8,10]$. Although we tried to find paralogs from the EST datasets to date the duplication, signals were mostly hidden or too weak. Finding recent whole genome duplication events using ESTs is somewhat difficult in that paralogous and redundant sequences are not clearly identifiable in EST datasets and recent whole genome duplication signals are frequently masked by recent single gene duplication signals. If the whole genome duplication occurred in Miscanthus after its divergence from a common ancestor shared with sorghum (ca. 6.2 MYA), two hypotheses are highly likely: (1) the Miscanthus genome had experienced some 'diploidization' (loss of one member of duplicated gene pairs) before the two Miscanthus species diverged (ca. 1.5 MYA), and (2) the (diploid) $M$. sinensis genome experienced appreciable diploidization after the two Miscanthus species diverged. If the former is true, M. sacchariflorus might have had an additional genome-wide duplication after the two species diverged. Considering the recent divergence of the two species (1.5 MYA), the latter may be more plausible. However, this has to be elucidated with additional information. 
Table 2 Putative functions of genes included in the three orthologous groups shown in Figure 4

\begin{tabular}{|c|c|c|c|c|c|c|}
\hline \multirow[b]{2}{*}{ Groups } & \multirow[b]{2}{*}{ DN \# } & \multicolumn{2}{|c|}{ M. sacchariflorus } & \multicolumn{2}{|c|}{ M. sinensis } & \multirow[b]{2}{*}{ Putative functions } \\
\hline & & Leaf $(A L)$ & Rhizome (AR) & Leaf (IL) & Rhizome (IR) & \\
\hline \multirow[t]{6}{*}{ I } & DN551695 & isotig00853 & isotig08093 & isotig00083 & isotig03753 & Polyubiquitin \\
\hline & DN552466 & isotig05764 & isotig07409 & isotig05877 & isotig08182 & Serine-threonine kinase receptor-associated protein \\
\hline & DN552491 & isotig03800 & isotig03933 & isotig09327 & isotig06471 & Heat shock protein 90 \\
\hline & DN552676 & isotig01244 & isotig07514 & isotig10039 & isotig01837 & kh domain containing protein \\
\hline & DN552708 & isotig05041 & isotig04880 & isotig07298 & isotig07578 & Catalase \\
\hline & DN552740 & isotig04698 & isotig00757 & isotig03722 & isotig02800 & rna-binding protein \\
\hline \multirow[t]{11}{*}{$\|$} & DN551779 & isotig00796 & isotig01314 & isotig01678 & isotig04296 & Amino acid expressed \\
\hline & DN551936 & isotig05246 & isotig06286 & isotig03192 & isotig09807 & Actin \\
\hline & DN552433 & isotig06434 & isotig05172 & isotig07270 & isotig07933 & at1g67350-like protein \\
\hline & DN552562 & isotig01135 & isotig05901 & isotig08694 & isotig08499 & Poly -binding protein \\
\hline & DN552570 & isotig06844 & isotig02287 & isotig05455 & isotig11299 & at5g02460-like protein \\
\hline & DN552671 & isotig06879 & isotig05162 & isotig09810 & isotig07725 & Plasminogen activator inhibitor 1 rna-binding protein \\
\hline & DN552679 & isotig02638 & isotig06563 & isotig01634 & isotig00900 & Acyl carrier protein 3 \\
\hline & DN552709 & isotig03948 & isotig03636 & isotig02927 & isotig02248 & Ras-related protein rab11c \\
\hline & DN552742 & isotig06246 & isotig03810 & isotig02007 & isotig05233 & No hits found \\
\hline & DN552766 & isotig02679 & isotig05804 & isotig03934 & isotig03115 & Membrane protein \\
\hline & DN552772 & isotig02777 & isotig06530 & isotig04172 & isotig09766 & S-adenosylmethionine decarboxylase \\
\hline \multirow[t]{14}{*}{ III } & DN551750 & isotig02250 & isotig03324 & isotig02111 & isotig04464 & Dolichyl-diphosphooligosaccharide-protein glycosyltransferase \\
\hline & DN551751 & isotig01665 & isotig00318 & isotig00973 & isotig00230 & 40s ribosomal protein s14 \\
\hline & DN551768 & isotig03736 & isotig03218 & isotig04157 & isotig01466 & No hits found \\
\hline & DN551788 & isotig05403 & isotig05029 & isotig05339 & isotig04880 & Reversibly glycosylated polypeptide \\
\hline & DN551793 & isotig00295 & isotig00036 & isotig00847 & isotig00643 & $60 \mathrm{~s}$ acidic ribosomal protein $\mathrm{p} 1$ \\
\hline & DN551798 & isotig00508 & isotig00357 & isotig02756 & isotig02156 & nc domain-containing protein \\
\hline & DN551841 & isotig07246 & isotig06497 & isotig04474 & isotig10222 & Maize proteinase inhibitor \\
\hline & DN551904 & isotig03373 & isotig04561 & isotig01269 & isotig04168 & Ubiquitin-like protein smt3 \\
\hline & DN552392 & isotig00059 & isotig01841 & isotig03321 & isotig00727 & No hits found \\
\hline & DN552427 & isotig07628 & isotig07842 & isotig01782 & isotig09528 & atp synthase gamma \\
\hline & DN552437 & isotig01515 & isotig04375 & isotig03437 & isotig02624 & Actin-depolymerizing factor 6 \\
\hline & DN552472 & isotig01693 & isotig01301 & isotig00608 & isotig00373 & nac2 protein \\
\hline & DN552626 & isotig01047 & isotig00215 & isotig00478 & isotig04878 & 60s ribosomal protein 126-1 \\
\hline & DN552650 & isotig07218 & isotig06635 & isotig04233 & isotig03259 & 40s ribosomal protein s15a \\
\hline
\end{tabular}

Groups indicate the three topologies of phylogenetic trees described in the figure. DN numbers indicate the NCBI accession numbers of rhizome-enriched genes from S. halepense or S. propinquum. Putative functions were predicted based on the best blastx hits of rhizome-enriched genes from S. halepense or S. propinquum against the NCBI's non-redundant protein database.

Functional differentiation of Miscanthus and sorghum genes Conserved protein domains are frequently essential to the function of a gene. Amino acid change that occurs in the conserved regions of a protein sequence is more likely to have larger impact on gene function than changes in other regions [20]. The impacts of nsSNPs on gene functions were predicted based on gene conservation profiles as described by Paterson et al. [21]. A total of 43,770 nsSNPs were identified between Miscanthus and sorghum and 35,818 nsSNPs between MSA and MSI. If a base involved in a specific nsSNP site is predominant in the 30 published genomes (see Materials and Methods), the base is defined as a common allele. Otherwise, it was defined as a rare allele. In Figure 6, the X-axis indicates the impact on gene function. If an nsSNP is changed from a common to a rare allele, it has positive value. Therefore, the impact of nsSNPs on gene function increases from negative to positive values. The Y-axis represents the probability of nsSNPs with different impact. The impacts on gene function of SNPs that differ between the two Miscanthus species are significantly more positive than SNPs between sorghum and 


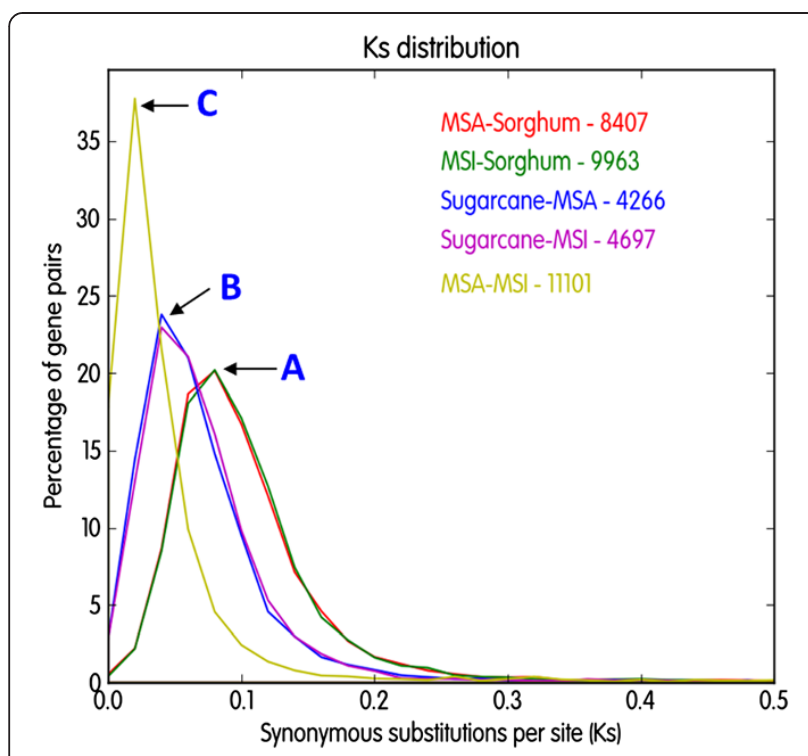

Figure 5 Distributions of synonymous nucleotide substitution (Ks) values among tested grasses. The numbers next to species names indicate the number of orthologous sequences used for plotting Ks distributions. Each peak represents time points of speciation between two species. (A) Speciation of two subtribes, Sorghinae and Saccarinae based on the comparison of MSA - sorghum and MSI - sorghum (Ks=0.08). (B) Speciation of Saccharum and Miscanthus genera based on MSA - sugarcane and MSI - sugarcane (Ks = 0.04). (C) Speciation between two Miscanthus species based on MSA-MSI (Ks=0.02). MSA; M. sacchariflorus, MSI; M. sinensis.
Miscanthus (t statistics $=125.37, P=0$ ), indicating that striking mutations are more frequently found between two Miscanthus species than sorghum and Miscanthus. This may be a consequence of the much higher level of gene duplication in Miscanthus, in which striking mutations may be better tolerated by virtue of the presence of a second gene copy that may confer essential functionality.

Table 3 shows the ten largest-effect nsSNPs that are found between reference (sorghum) and mutated alleles (Miscanthus). The ten genes could be classified into five functional categories which are $\mathrm{ABC}$ transporters, glycogen synthase kinase-3, ATPase, beta tubulin, and cellulose synthase. Interestingly, except for cellulose synthase (Sb01g019720), three functions (ABC transporter, ATPase, and glycogen synthase kinase-3) and beta tubulin have very close GO terms, ATP binding (GO:0005524) and GTP binding (GO:0005525), respectively. These genes may be of interest for further functional investigation.

\section{Conclusions}

The current study provides nearly 227,000 ESTs and 146,000 ESTs from M. sinensis and M. sacchariflorus, respectively, greatly enriching knowledge of the transcriptome of this promising lignocellulosic bioethanol crop. M. sinensis and M. sacchariflorus are of particular interest because they are suspected progenitors of the triploid M. $\times$ giganteus which is cultivated as a biomass crop. These ESTs can be utilized in many ways such as seeking molecular markers, gene prediction in

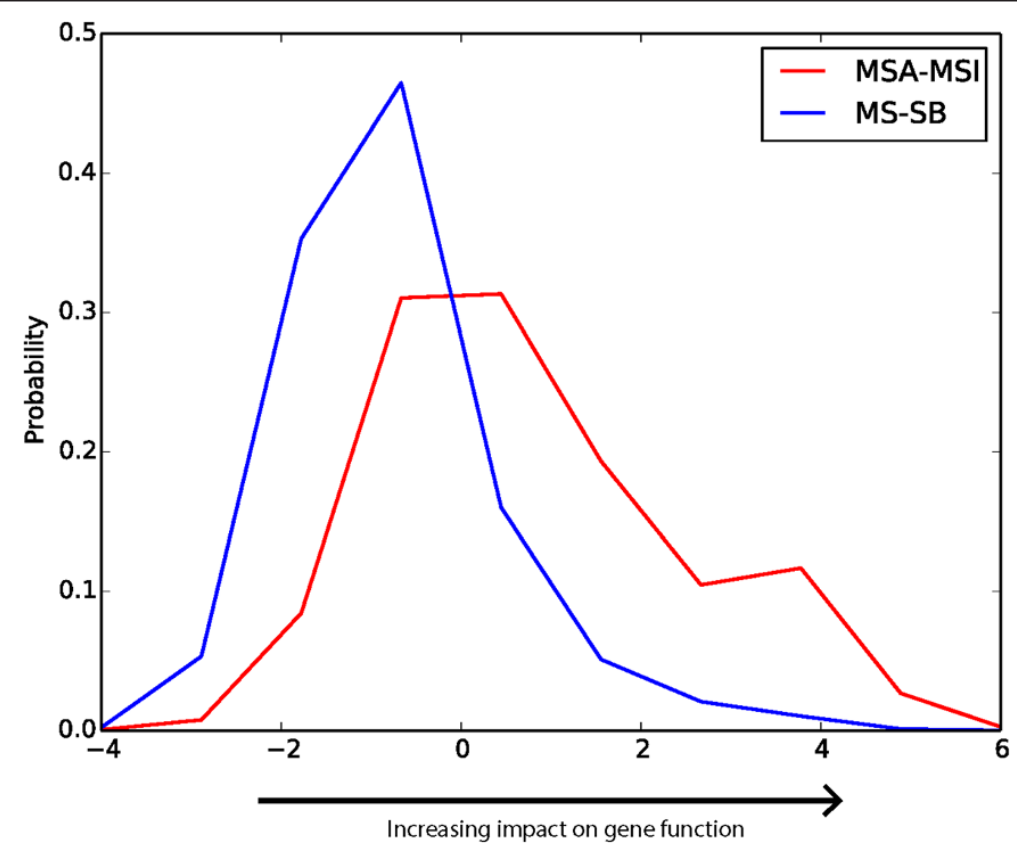

Figure 6 Distribution of nsSNP impact on protein function in the sorghum and Miscanthus lineages. Red line represents nsSNP between the two Miscanthus species. Blue line depicts nsSNPs between Miscanthus and sorghum. 
Table 3 Top 10 nsSNPs having large impacts on gene functions

\begin{tabular}{|c|c|c|c|c|}
\hline Gene & Position & Ref AA & Mut AA & Function \\
\hline Sb10g004070 & 691 & W & $\mathrm{H}$ & $\begin{array}{l}\text { Pleiotropic drug resistance } \\
\text { proteins, } A B C \text { superfamily }\end{array}$ \\
\hline Sb01g043620 & 323 & V & । & Calcium transporting ATPase \\
\hline Sb01g019720 & 694 & E & Q & $\begin{array}{l}\text { C1-like domain|Cellulose } \\
\text { synthase }\end{array}$ \\
\hline Sb01g006310 & 193 & V & । & Beta tubulin \\
\hline Sb04g008580 & 231 & G & S & Glycogen synthase kinase-3 \\
\hline Sb10g004070 & 688 & Q & S & $\begin{array}{l}\text { Pleiotropic drug resistance } \\
\text { proteins, } A B C \text { superfamily }\end{array}$ \\
\hline Sb03g027480 & 801 & M & । & $\begin{array}{l}\text { Pleiotropic drug resistance } \\
\text { proteins, } A B C \text { superfamily }\end{array}$ \\
\hline Sb03g027490 & 849 & M & । & $\begin{array}{l}\text { Pleiotropic drug resistance } \\
\text { proteins, } A B C \text { superfamily }\end{array}$ \\
\hline Sb04g008580 & 252 & A & E & Glycogen synthase kinase-3 \\
\hline Sb10g004070 & 692 & । & G & $\begin{array}{l}\text { Pleiotropic drug resistance } \\
\text { proteins, } A B C \text { superfamily }\end{array}$ \\
\hline
\end{tabular}

Reference (Ref AA) and mutation amino acid (Mut AA) indicate sorghum allele and mutated allele in Miscanthus, respectively.

genome sequences, and gene expression studies. A core set of (largely housekeeping) genes are expressed in common in the two Miscanthus species and the two tissues studied, with large populations of genes that appear to be tissue specific. Genes showing rhizome-enriched expression in rhizomatous Sorghum species appear to correspond more closely to those in $M$. sacchariflorus which has aggressive rhizomes, than those in $M$. sinensis that has weak rhizomes. The analysis of nsSNPs showed that striking mutations are more frequently found between two Miscanthus species than between Miscanthus and sorghum, reflect that Miscanthus seems to be more tolerant of point mutations than sorghum. A plausible explanation of this observation is that genomic redundancy resulting from its recent genome doubling has relaxed selection on duplicated gene copies. We also analyzed ESTs to make preliminary inferences about Miscanthus evolution. As stated in the text, Miscanthus experienced whole genome duplication since its divergence from sorghum. Although ESTs generally provided too little information to clearly identify paralogs which were formed by the whole genome duplication, they were still useful to predict speciation events by providing clear signal of orthologous relationships. The exact timing of the whole-genome duplication event can be evaluated when additional genome sequence and detailed maps are available, and our ESTs will be able to contribute to such detailed evolutionary studies.

\section{Methods}

\section{Plant materials and growth conditions}

M. sinensis and M. sacchariflorus were collected in South Korea, and their ploidy levels and morphological identities were determined as in a previous report [22]. Plants were grown for a year in plastic pots and placed in the greenhouse at Mokpo National University, Mokpo, South Korea. No fertilizer was applied until sampling, but watering was given when the pot soil dried. The greenhouse temperature was controlled from 24 to $28^{\circ} \mathrm{C}$ during the growth period without supplemental light. Leaves and rhizomes were sampled in the vegetative state before flowering. Newly developed rhizomes ( $<1$ year old) along inner surface of the pot were harvested and pooled after grinding. All samples were sent to the National Instrumentation Center for Environmental Management (NICEM, Seoul, South Korea) for cDNA library construction.

\section{Construction of CDNA library and sequencing of the library}

Total RNA was isolated from rhizome and leaf tissues of M. sinensis and M. sacchariflorus using Rneasy Plant Mini Kit (Qiagen, Seoul, Korea) according to the manufacturer's instructions. Extracted RNA samples were quantified and quality-checked using a Bioanalyzer 2100 (Agilent Technologies, Santa Clara, CA). Total RNA (6 $\mu \mathrm{g})$ was reverse transcribed using Super Script II (Life Technologies, Carlsbad, CA). Second strand cDNA was synthesized using Advantage 2 Polymerase Mix (Clontech, Seoul, Korea). cDNA samples were purified using QIAquick PCR purification kit (Qiagen, Seoul, Korea). The purified fragments were then used to create the 454 single-stranded cDNA library using a 454 library preparation kit (Roche). The fragment ends were polished using T4 ligase and T4 polynucleotide kinase and adaptors containing primer sequences and a biotin tag were ligated to the fragment ends (Roche, Brandford, CT). The fragments with properly ligated adaptors were immobilized onto magnetic streptavidincoated beads (Roche, Brandford, CT). Nicks or gaps between the adaptors and the dscDNA fragments were repaired using the fill-in polymerase. The non-biotinylated strands of the immobilized dscDNA fragments were melted off to generate the single-stranded cDNA library for 454 sequencing.

\section{Assembly of 454 reads and annotation of contigs}

Before assembly, extended multiplex identifier (MID) sequences of $3^{\prime}$ and $5^{\prime}$ ends, poly $\mathrm{A}(\mathrm{T})$ tail, short sequences ( $<50 \mathrm{bp})$ and low complexity were removed. Four EST data sets from leaf and rhizome tissues of two Miscanthus species were individually assembled using GS de novo assembler version 2.6 (Roche Diagnostics Corporation, 454.com/ products/analysis-software/index.asp) with default parameters. From the assembly results, only consensus sequences (contigs) were taken for further analyses due to the short length of singletons. Functional annotation was performed by sequence comparison with public databases. All contigs were blasted against the Phytozome database (www.phytozome.org, version 7.0, April 2011) 
which includes annotated genes with the KOG assignments $\left(e<10^{-5}\right)$.

\section{Comparison to rhizome-enriched genes from S. halepense and S. propinquum}

A total of 768 ESTs previously reported as derived from rhizome-enriched genes in S. halepense or S. propinquum [11] were blasted against Miscanthus ESTs to find putative orthologs $\left(e<10^{-20}\right)$. The putative orthologs from five different datasets - rhizome and leaf ESTs from two Miscanthus species and rhizome-enriched genes from Sorghum spp. - were used to construct phylogenetic trees. The five orthologs were multiple-aligned by ClustalW [23]. ClustalW was also implemented for generating phylogenetic trees using the Neighbor Joining algorithm. The phylogenetic trees were grouped based on their topologies by determining symmetric differences among trees with the Treedist function in the PHYLIP package [24], in order to compare rhizome-enriched genes from the two Sorghum species to ESTs from Miscanthus. The phylogenetic trees were grouped based on their topologies, in order to infer rhizome-enriched ESTs in Miscanthus species. For the candidate rhizome-enriched ESTs in Miscanthus, putative functions were classified using Blast2GO [25].

\section{Estimation of divergence time in Saccharinae subtribe}

To estimate the speciation time among $M$. sacchariflorus, M. sinensis, sugarcane, and sorghum, Ks values between orthologous ESTs were calculated. Sugarcane ESTs and sorghum gene sequences were obtained from dbEST (http://www.ncbi.nlm.nih.gov/dbEST/) and Phytozome (www.phytozome.net/), respectively. To identify putative orthologs among species, each sequence from one species was blasted against all sequences from other species and the best hits were taken. A pair of best hit was defined as putative orthologs when the pair was aligned over $300 \mathrm{bp}$ or more $\left(e<10^{-20}\right)$. Each member of a pair of sequences was subjected to blastx search against predicted sorghum proteins. The best match was considered significant if the alignment length was more than 100 amino acids $\left(e<10^{-15}\right)$. If no significant match was found, the pair of sequences was excluded from further analysis. The cleaned pairs of sequences were translated using the Genewise program, which can take frameshift sites into consideration [26], with the corresponding best match protein in S. bicolor as reference. For each pair of paralogs, the two translated products were aligned using ClustalW [23], and the resulting alignment was used as a guide to align the nucleotide sequences. After removing gaps and $\mathrm{N}$-containing codons, the level of synonymous substitution was estimated using the maximum likelihood approach implemented in the program CODEML, which is a part of the PAML package [27]. Batch jobs were performed using in-house python scripts.

\section{Nonsynonymous SNP analysis}

Orthologous protein sequences from S. bicolor, $M$. sacchariflorus and $M$. sinensis previously defined were used to identify nsSNP. To find nsSNPs between MSA and MSI, pairwise alignment of predicted proteins were conducted. For nsSNPs between sorghum and Miscanthus, the exactly same proteins across the two Miscanthus species were aligned to sorghum proteins. The identified nsSNPs were classified into two groups: One group contains nsSNP that is polymorphic between MSA and MSI. nsSNPs in the other group are between sorghum and the two Miscanthus species. Protein sequences from the 30 published genomes (http://chibba.agtec.uga.edu/duplication/) were clustered and aligned using orthoMCL [28] and ClustalW [23]. The nsSNPs identified from the three species were aligned to the orthologous gene clusters. The impact of nsSNPs on gene function is evaluated by functional impact score (FIS) using the formula described by Paterson et al. [21].

\section{Availability}

All the ESTs are downloadable at the NABIC (National Agricultural Biotechnology Information Center; http:// nabic.rda.go.kr/) with accession numbers indicated; NN-0642-000001 (MSAL), NN-0643-000001 (MSAR), NN-0639-000001 (MSIL), and NN-0641-000001 (MSIR).

\begin{abstract}
Abbreviations
AFLP: Amplified fragment length polymorphism; SSR: Simple sequence repeat; EST: Expressed sequence tag; MYA: Million years ago; MID: Multiplex IDentifier; GO: Gene ontology; MSI: M. sinensis; MSA: M. sacchariflorus; MSIL: $M$. sinensis leaf library; MSIR: $M$. sinensis rhizome library; MSAL: M. sacchariflorus leaf library; MSAR: $M$. sacchariflorus rhizome library; nsSNP: Nonsynonymous single nucleotide polymorphism; Ks: Synonymous nucleotide substitution.
\end{abstract}

\section{Competing interests}

The authors declare that they have no competing interests.

\section{Authors' contributions}

AHP, DSK, GJL conceived the study. CK, THL, AHP, GJL analyzed results and wrote the paper. YJJ, SJC performed experiments. DSK provided materials. THL, HG provided bioinformatics support and discussion. All authors read and approved the final manuscript.

\section{Acknowledgement}

This work was supported by a grant from the Plant Molecular Breeding Center of the Next-Generation BioGreen 21 Program, RDA (grant no. PJ0081312012); the US Department of Energy-US Department of Agriculture Plant Feedstock Program (project grant number: 112786); the Consortium for Plant Biotechnology Research (CPBR); the National Science Foundation (NSF: DBI 0849896); and resources and technical expertise from the University of Georgia, Georgia Advanced Computing Resource Center, a partnership between the Office of the Vice President for Research and the Office of the Chief Information Officer.

\section{Author details}

${ }^{1}$ Plant Genome Mapping Lab, University of Georgia, 111 Riverbend RD, Athens, GA 30602, USA. ²Department of Horticulture, Chungnam National University, Daejeon 305-764, South Korea. ${ }^{3}$ Department of Plant Science, Research Institute of Agriculture and Life Sciences, Seoul National University, 
Seoul 151-921, South Korea. ${ }^{4}$ Present address: Genomics Division, National Academy of Agricultural Science, Rural Development Administration, Suwon 441-707, South Korea.

Received: 5 February 2014 Accepted: 13 May 2014

Published: 18 May 2014

\section{References}

1. Boehmel C, Lewandowski I, Claupein W: Comparing annual and perennial energy cropping systems with different management intensities. Agr Syst 2008, 96(1-3):224-236.

2. Dohleman FG, Long SP: More productive than maize in the Midwest: How does Miscanthus do it? Plant Physiol 2009, 150(4):2104-2115.

3. Wang J, Roe B, Macmil S, Yu Q, Murray JE, Tang H, Chen C, Najar F, Wiley G, Bowers J, Van Sluys MA, Rokhsar DS, Hudson ME, Moose SP, Paterson AH, Ming R: Microcollinearity between autopolyploid sugarcane and diploid sorghum genomes. BMC Genomics 2010, 11:261.

4. Hodkinson TR, Chase MW, Lledo MD, Salamin N, Renvoize SA: Phylogenetics of Miscanthus, Saccharum and related genera (Saccharinae, Andropogoneae, Poaceae) based on DNA sequences from ITS nuclear ribosomal DNA and plasted trnL intron and trnL-F intergenic spacers. J Plant Res 2002, 115:381-392.

5. Sacks EJ, Juvik JA, Lin Q, Stewart R, Yamada T: The Gene Pool Of Miscanthus Species And Its Improvement. In Genomics of the Saccharinae. Edited by Paterson AH. New York: Springer; 2013:73-101.

6. Paterson AH, Bowers JE, Bruggmann R, Dubchak I, Grimwood J, Gundlach H, Haberer G, Hellsten U, Miltros T, Poliakov A, Schmutz J, Spannagl M, Tang H, Wang X, Wicker T, Bharti AK, Chapman J, Feltus FA, Gowik U, Grigoriev IV, Lyons E, Maher CA, Martis M, Narechania A, Otillar RP, Penning BW, Salamov AA, Wang Y, Zhang L, Carpita NC, et al: The Sorghum bicolor genome and the diversification of grasses. Nature 2009, 457:551-556.

7. Atienza SG, Satovic Z, Peterson KK, Dolstra O: Preliminary genetic linkage map of Miscanthus sinensis with RAPD markers. Theor Appl Genet 2002, 105:946-952.

8. Kim C, Zhang D, Auckland SA, Rainville LK, Jakob K, Kronmiller B, Sacks EJ, Deuter M, Paterson AH: SSR-based genetic maps of Miscanthus sinensis and M. sacchariflorus, and their comparison to sorghum. Theor Appl Genet 2012, 124(7):1325-1338.

9. Swaminathan $\mathrm{K}$, Chae WB, Mitros T, Varala K, Xie L, Barling A, Glowacha K, Hall M, Jezowski S, Ming R, Hudson M, Juvik JA, Rokhsar DS, Moose SP: A framework genetic map for Miscanthus sinensis from RNAseq-based markers shows recent tetraploidy. BMC Genomics 2012, 13:142.

10. Ma XF, Jensen E, Alexandrov N, Troukhan M, Zhang L, Thomas-Jones S, Farrar K, Clifton-Brown J, Donnison I, Swaller T, Flavell R: High resolution genetic mapping by genome sequencing reveals genome duplication and tetraploid genetic structure of the diploid Miscanthus sinensis. PLoS One 2012, 7(3):e33821.

11. Jang CS, Kamps TL, Skinner DN, Schulze SR, Vencill WK, Paterson AH: Functional classification, genomic organization, putatively cis-acting regulatory elements, and relationship to quantitative trait loci, of sorghum genes with rhizome-enriched expression. Plant Physiol 2006, 142(3):1148-1159.

12. Rayburn AL, Crawford J, Rayburn C, Juvik J: Genome size of three Miscanthus species. Plant Mol Biol Rep 2009, 27(2):184-188.

13. Goodstein DM, Shu S, Howson R, Neupane R, Hayes RD, Fazo J, Mitros T, Dirks W, Hellsten U, Putnam N, Rokhsar DS: Phytozome: a comparative platform for green plant genomics. Nucleic Acids Res 2012, 40(Database issue):D1178-1186

14. Tatusov RL, Fedorova ND, Jackson JD, Jacobs AR, Kiryutin B, Koonin EV, Krylov DM, Mazumder R, Mekhedov SL, Nikolskaya AN, Rao BS, Smirnov S, Sverdlov AV, Vasudevan S, Wolf YI, Yin JJ, Natale DA: The COG database: an updated version includes eukaryotes. BMC Bioinforma 2003, 4:41.

15. Jang CS, Kamps TL, Tang H, Bowers JE, Lemke C, Paterson AH: Evolutionary fate of rhizome-specific genes in a non-rhizomatous Sorghum genotype. Heredity 2009, 102(3):266-273.

16. Gaut BS, Morton BR, McCaig BC, Clegg MT: Substitution rate comparisons between grasses and palms: Synonymous rate differences at the nuclear gene Adh parallel rate differences at the plastid gene rbcL. Proc Natl Acad Sci U S A 1996, 93:10274-10279.

17. Jannoo N, Grivet L, Chantret N, Garsmeur O, Glaszmann J-C, Arruda P, D'Hont A: Orthologous comparison in a gene-rich region among grasses reveals stability in the sugarcane polyploid genome. Plant J 2007, 50:574-585.
18. Kim C, Tang H, Paterson AH: Duplication and divergence of grass genomes: Integrating the Cloridoids. Tropical Plant Biol 2009, 2:51-62.

19. Berding N, Roach BT: Germplasm collection, Maintenence, and Use. In Sugarcane Improvement Through Breeding. Edited by Heinz DJ. Amsterdam: Elsevier Press; 1987:143-210.

20. Choi Y, Sims GE, Murphy S, Miller JR, Chan AP: Predicting the functional effect of amino acid substitutions and indels. PLoS One 2012, 7(10):e46688.

21. Paterson AH, Wendel JF, Gundlach H, Guo H, Jenkins J, Jin D, Llewellyn D, Showmaker KC, Shu S, Udall J, Yoo MJ, Byers R, Chen W, Doron-Faigenboim A, Duke MV, Gong L, Grimwood J, Grover C, Grupp K, Hu G, Lee TH, Li J, Lin L, Liu T, Marler BS, Page JT, Roberts AW, Romanel E, Sanders WS, Szadkowski E, et al: Repeated polyploidization of Gossypium genomes and the evolution of spinnable cotton fibres. Nature 2012, 492(7429):423-427.

22. Lee KY, Zhang L, Lee G-J: Botanical and germinating characteristics of Miscanthus species native to Korea. Hortic Environ Biotechnol 2012. 53:490-496.

23. Thompson JD, Higgins DG, Gibson TJ: CLUSTAL W: improving the sensitivity of progressive multiple sequence alignment through sequence weighting, position-specific gap penalties and weight matrix choice. Nucleic Acids Res 1994, 22(22):4673-4680.

24. Felsenstein J: PHYLIP - Phylogeny Inference Package (Version 3.2). Cladistics 1989, 5(2):163-166.

25. Conesa A, Gotz S, Garcia-Gomez JM, Terol J, Talon M, Robles M: Blast2GO: a universal tool for annotation, visualization and analysis in functional genomics research. Bioinformatics 2005, 21(18):3674-3676.

26. Birney E: Genewise and genomewise. Genome Res 2004, 14(5):988-995.

27. Yang Z: PAML 4: phylogenetic analysis by maximum likelihood. Mol Biol Evol 2007, 24(8):1586-1591.

28. Li L, Stoeckert CJ Jr, Roos DS: OrthoMCL: identification of ortholog groups for eukaryotic genomes. Genome Res 2003, 13(9):2178-2189.

doi:10.1186/1471-2229-14-134

Cite this article as: Kim et al:: Sequencing of transcriptomes from two

Miscanthus species reveals functional specificity in rhizomes, and clarifies evolutionary relationships. BMC Plant Biology 2014 14:134.

\section{Submit your next manuscript to BioMed Central and take full advantage of:}

- Convenient online submission

- Thorough peer review

- No space constraints or color figure charges

- Immediate publication on acceptance

- Inclusion in PubMed, CAS, Scopus and Google Scholar

- Research which is freely available for redistribution 